\section{Laser-Molecule Interaction}

Jean Rene Lalanne, Andre Ducasse, and Stanislaw Kielich, 330 pages, illustrations, index, references, and appendices. ISBN 2-84054-017-8. John Wiley \& Sons, Inc., 605 Third Avenue, New York, NY (1996) $\$ 59.95$ hardbound.

Reviewed by Roger J. Becker, University of Dayton, Research Institute, KL-545, Dayton, OH 45469-0150.

This text is billed as integrating coverage of the fundamentals of laser science with an introduction to nonlinear optics on the molecular level. From the standpoint of traditional course synopses, it may, with some charity, be more properly viewed as extending a solid introduction to laser science to give an excellent background for a later course in nonlinear optics. The level is best suited for a second-year graduate student with a prior course in quantum mechanics.

Most graduate level texts tend to be rather dry, especially in the case of quantum optics. To their credit the authors and their editor have expended considerable effort to put some zest into their several subjects. Albeit presented at an advanced level, the book's major strength is its accessibility. Its approach is student friendly, with a generous use of illustrations and a pedagogical treatment highlighting major points and reviewing key concepts.

Nearly three dozen worked examples are presented and used to introduce auxiliary topics, which stimulate reader interest. Good emphasis is placed on the essential physics governing various phenomena. For example, the impact of diffraction on wave propagation in a laser cavity is highlighted as the reason for the ubiquity of Gaussian optics in many lasers. This is a saving grace; the fundamental weakness of the book is its brevity in connection with its attempt to cover a wide range of subjects. By spreading themselves too thin, the authors seriously impair the competitiveness of the work in comparison with a standard text addressing a traditional course structure. Using the same example, the authors use only about one tenth of the space devoted by Verdeyen [Laser Electronics, 3rd ed., Prentice Hall, Englewood Cliffs, NJ (1995)] to Gaussian beams and laser optics. Thus the mode structure of laser cavities is not made very clear. Another example is the treatment of the density matrix, a major component of the structure on which the book is based. The author's treatment is much shorter than Verdeyen's. This terseness makes much of the material abstract.

Much of the subject matter is presented in the examples, so that they lose potential for clarifying material discussed earlier as text. An example on Q-switching is unsupported with any discussion of the $\mathrm{Q}$ of a cavity and only a fairly terse treatment of Q-spoiling. Depending on the perspicacity of the student, this compression may be viewed either as a success in efficient editing or as a recipe for bewilderment. Evidently the extensive use of supplemental material is assumed.

The first three chapters review quantum mechanics, tensors, and some essentials of statistical mechanics. This treatment includes a good introduction to the density matrix, which is used in later material. The discussion on tensors for the major point groups is excellent. The attempt to give a self-contained treatment of quantum mechanics is clearly tongue in cheek. Advanced topics are presented without significant time to absorb fundamental ones. For instance "Dyson's equation" appears on page 12 . The postulates of quantum mechanics, time evolution operators, and state vectors are covered in 13 pages. This can hardly be considered an introduction to quantum mechanics, and does not constitute a very comprehensive review. Similarly the treatment on statistical mechanics is really a review of the taking of averages, which is used later in the text. There is simply not space enough to introduce these subjects and as review they are in my judgment largely superfluous.

The bulk of the work covers laser theory and as an introduction is adequate for a graduate course, although efficiency, noise, hole burning, and Raman shifting are not covered. One nice nugget is an emphasis on the various time scales affecting the temporal characteristics of a laser. Ring lasers are given good coverage. Materials' properties, such as semiconductor and gas discharge physics, are not addressed. The authors thus miss a chance to capitalize on their earlier treatment of symmetry.

Although only two chapters are nominally devoted to nonlinear optics, many topics are addressed in earlier chapters. Thus the book does succeed in its aim of integrating the various subjects addressed. However, virtually all of the topics addressed, save for the discussion of tensors and the symmetry properties of point groups, could be considered as part of a laser course and are covered in Siegman [Lasers, University Science Books, Mill Valley (1986)]. Almost all of them are included in Verdeyen. So what we have is really a laser book. Moreover, while exotic topics such as atom cooling, Ramsey fringes, photon echoes, and quantum beats are discussed, fundamentals of nonlinear optics that are not covered include dispersion, parametric mixing, four-wave mixing, stimulated Raman and Brillouin scattering, wave propagation, and mode coupling, to say nothing of devices, fiber optics, and solitons. Oddly, for a text initially directed at chemical physicists, only a terse treatment of bonding and materials is presented.

The book is translated from a French edition, and many passages are stilted. Operators are not given any distinguishing font Against these minor blemishes, the layout of the text is superb. In all, the effort to provide a highly readable text is one of the most compelling advantages of the work.

In conclusion, Laser-Molecule Interaction is essentially an introductory course in laser physics. It does not cover either quantum optics or nonlinear optics in a comprehensive manner. It would have been preferable if the authors had focused on laser electronics. As it is, the work would serve well for review by a bright student or as supplementary reading.

\section{Principles and Applications of Optical Communications}

Max Ming-Kang Liu, xix +1004 pages, illustrations, index, references, and five appendices. ISBN: 0-256-16415-0. Richard D. Irwin, Inc., 1333 Burr Ridge Parkway, Burr Ridge, Illinois, 60521-0085 (1996) \$55 hardbound.

Reviewed by Mohammad F. Mahmood, Computational Science and Engineering Research Center, Howard University, Washington, D.C. 20059.

This book, for students at the first-year graduate level, is intended to serve as an introduction without the detailed analytical 
emphasis common in other texts on optical communications. Throughout, the emphasis is placed on physical and heuristic descriptions supplemented by extensive solved examples with minimal mathematical and theoretical treatment of the subject. A number of exercises also appear at the end of each chapter to help the reader develop a better understanding of the subject material. Because it is an introductory book, essential material from one chapter is summarized in another to make each chapter self-contained and to minimize the need for reference to other work. For the same reason, the references have been limited to papers of historical importance. The book is arranged into 18 chapters grouped into four parts: overview (Chaps. 1 and 2), basic communication blocks (Chaps. 3 to 7), networking (Chaps. 8 to 11 ), and signal processing (Chaps. 12 to 18). This is followed by five useful appendices.

Part one starts with a review of recent developments in lightwave technology throughout the last two decades with reference to communication systems and communication networks. It describes basic building blocks in the communication process and addresses some design issues in optical communications. It also explains important networking concepts and introduces some network designs to improve the overall throughput.

Part two deals with the description of various light sources in optical communications, optical fibers, light detection, noise in optical communications, and incoherent detection. To gain a good understanding of the subject material covered in this part, the reader is assumed to have some background in electromagnetic theory; some understanding of optics and quantum physics; and some familiarity with the mathematical probability. This part also serves as a foundation for subsequent discussion in the remaining two parts.

Part three focuses on the basic principles of time-domain medium access, wavelength-domain medium access, subcarrier multiplexing, and photonic switching. This part basically explains various multiplexing and switching techniques in optical communication networks.

The final part is devoted to some advanced topics in optical communications such as direct modulation, distributed feedback laser diodes and modulation, external modulation, coherent detection, timing recovery and line coding, optical amplification, and optical fiber soliton transmission. It helps readers to visualize detection schemes and timing recovery techniques in high-speed digital communications. The author also gives a fairly short but interesting discussion on the subject of light signal transmission, recent technology developments on optical amplifiers, and optical solitons.
Although every effort has been made to present the subject matter in a coherent way, the book to me represents some sort of compromise. In some cases, for instance, only the basic features of a problem are discussed, with very little detail given. But for the most part, the book is well written and easy to follow. In all, I have found this book an excellent introductory text on the important and rapidly growing field of optical communication.

\section{BOOKS RECEIVED}

Photonics Rules of Thumb: Optics, Electro-Optics, Fiber Optics, by John Lester Miller and Ed Friedman. xiv +476 pp., illus., subject index, references following each chapter, bibliography. From the Optical and Electro-Optical Engineering Series. ISBN 0-07-044329-7. McGraw Hill, 11 West 19th Street, New York, NY 10011 (1996) \$39 hardbound. For quick and easy reference, this is a handy compilation of 250 rules of thumb that cover the full range of photonics, from optics to lasers.

Principles of High-Resolution Radar, by August W. Rihaczek. iii+498 pp., illus., subject index, bibliography, list of principal symbols, references following each chapter. From the Artech House Radar Library. ISBN 0-89006-900-X. Artech House, Inc., 685 Canton Street, Norwood, MA 02062 (1996) \$75 hardbound. Topics include fundamentals of waveform analysis; singletarget measurements; resolution in a matched-filter radar; resolution theory for targets with constant range rate; pulse compression waveforms; linear FM waveforms; coherent pulse trains; radar mapping of distributed targets; target detection in clutter.

Glasses for Infrared Optics, by Valentina F. Kokorina. 236 pp., illus., subject index, author bibliography, references at end of book. From the CRC Press Laser and Optical Science and Technology Series. ISBN 0-8493-3785-2. CRC Press, Inc., 2000 Corporate Blvd. N.W., Boca Raton, FL 33431 (1996) \$89.95 hardbound. Covers: chalcogenide glasses-a peculiar class of vitreous substances; optical properties of chalcogenide glasses; elaboration of commercial glasses; and technological basics for manufacturing optical chalcogenide glasses.

Handbook of Atmospheric Electrodynamics, Volumes I and II, edited by Hans Volland. 408 pp. in Vol. I, 516 pp. in Vol. II, illus., subject index, references following each paper. ISBN: Vol. I 0-84938647-0, Vol. II 0-8493-2520-X. CRC Press, Inc., 2000 Corporate Blvd. N.W.,
Boca Raton, FL 33431 (1996) Vol. I: \$129.95 hardbound; Vol. II: \$139.95 hardbound. This handbook is the extension of the two-volume CRC Handbook of Atmospherics, published in 1982, which covered only geoelectricity, with particular emphasis on lightning and sferics phenomena. The present handbook updates the 1982 edition and also includes low-frequency electric and magnetic fields and currents in the ionosphere and magnetosphere.

Introduction to Fourier Optics: Second Edition, by Joseph W. Goodman. xviii +441 pp., illus., subject index, problems following each chapter, bibliography at end of book. From the McGraw-Hill Series in Electrical and Computer Engineering. ISBN 0-07-024254-2. McGraw Hill, 1 West 19th Street, New York, NY 10011 (1996) \$96.25 hardbound. Expands the original edition published in 1968. Explores the applications of Fourier analysis in the field of optics with special emphasis on applications to diffraction, imaging, optical data processing, and holography.

Real-Time Optical Information Processing, edited by Bahram Javidi and Joseph L. Horner. $x+536$ pp., illus., subject index, references following each chapter, list of contributors. ISBN 0-12-381180-5. Academic Press, Inc., 525 B Street, Suite 1900 , San Diego, CA 92101-4495 (1994) \$99 hardbound. Presents recent advances in optical information processing, pattern recognition, neural computing, and materials for devices in optical computing. Significant signal processing algorithms and architectures in optics as well as basic hardware concepts such as the fundamentals of spatial light modulators are covered. Each chapter begins with a review of the basic concepts and follows with a discussion of recent advances in the field.

Handbook of Recording Engineering: Third Edition, by John M. Eargle. $\mathrm{x}+536$ pp., illus., subject index, bibliography following each section. ISBN 0-412-09741-9. Van Nostrand Reinhold, 115 Fifth Avenue, New York, NY 10003 (1996) \$59.95 hardbound. Expands on material from the second edition, including a complete revision of chapters on digital recording and signal processing, in addition to thorough coverage of analog tape recording and codedecode noise-reduction systems. Recording systems are examined in detail including discussion of digital consoles and digital metering as well as the monitoring environment. 